\title{
Reference Model for Operation and Reconfiguration of a Network for Subway Construction
}

\author{
Larissa Araújo ${ }^{1}$ and Fábio Müller Guerrini² \\ ${ }^{1}$ UFRN, Production Engineering Department, Av. Sen. Salgado Filho, 3000, \\ 59072 - 970 Natal/RN, Brazil \\ larissa@ct.ufrn.br \\ ${ }^{2}$ EESC/USP, Production Engineering Department, Av. Trabalhador são-carlense, 400, \\ 13566-590, São Carlos/SP, Brazil \\ guerriniasc.usp.br
}

\begin{abstract}
The Construction is an industry that depends on interorganizational relationships to enable its product. Most of the projects are held from networks, especially those for large scale projects of infrastructure, with flexible relationships for cooperation, and the modus operandi of virtual enterprises. However, there is a lack of studies on the analytical approach of networks considered their life cycle and the operation and reconfiguration phases for these large projects. The paper aims at developing a Reference Model for the phases of operation and reconfiguration of a construction network, specifically a subway infrastructure project. The model used a development process and is represented with the EKD Methodology (Enterprise Knowledge Development). A Case Study was used as reference. The Model enables the coordination of network activities from the perspective of collaboration. Other research opportunities emerge aiming at obtaining advances in scope and scale, considering this Case Study as an initial effort.
\end{abstract}

Keywords: construction, operation, reconfiguration, collaborative networks.

\section{Introduction}

Construction projects are inherently complex and dynamic, especially when considering the execution or operation phase in which a highly fragmented supply chain takes place [1], [2]. For large scale projects this complexity is also increased, demanding an extended network of people and resources within various functions and organizations. But the actual project management held by the construction industry is still traditional and leads to an environment with conflicts and wastes, the reason to consider the sector as inefficient [3].

Most of the managerial developments addressed to the construction industry are isolated initiatives. These initiatives are very relevant, but they do not have a systematic capacity to solve the managerial problems faced by the construction industry - most of the projects are unique with a great number of unpredictable events [4]. If we also consider the shortened time extensions and limited budgets [5] this capacity is even reduced, claiming to more systematic and integrative approaches.

Collaborative Networks have emerged in a great variety of ways [6], [7] and these value added alliances are becoming key ingredients to success, with the construction 
sector having a strong potential to benefit from the adoption of these new business modes of operation [8]. Also, adaptable and rapidly changing information and communication technologies (ICTs) support new patterns of work, focusing on crossdisciplinary, collaborative work [5], [8]. Moreover, the support of ICTs encourages the use of virtual business modes of operation as a result of a desire to improve market position, gain competitive advantage, and the need to add value [9]. The Virtual Enterprise (VE) concept is as a form of Collaborative Network - briefly described as a temporary alliance of enterprises supported by computer networks [6].

This temporary aspect is the main driver to make the association of a Construction Project with a Virtual Enterprise. It is important to emphasize that "in fact, the Construction industry has adopted the modus operandi of a VE for decades" [8]. The main insight gained from this association is to consider that both have a delimited Life Cycle, in which there are different phases that should be carefully considered when wishing to manage them effectively [5], [7]. For [7], the phases of a Network Life Cycle are: creation, operation, evolution and dissolution.

In this context, this paper aims at developing a Reference Model for the phases of operation and reconfiguration of a construction network, specifically a subway infrastructure project. Through the next sessions the literature review, the development methodology, the Data Collection and the Reference Model developed are discussed, followed by the Conclusions.

\section{$2 \quad$ Literature Review}

To conduct this research some topics of interest are necessary to serve as conceptual discussion for the reference model of the construction network.

Operation and Reconfiguration. The classic business phase "produce" in the construction case is "construct". The operation phase is the one characterized by the value generation, the consolidation of the business project initialized.

The reconfiguration or evolution happens because the initial deals are not maintained through the time (due to non predictable events), so they should continuously be revised. The initial partners will be reorganized, to maintain the relationships - the comprehension and proximity between these partners is of special importance [10]. The operation phase is considered the most important one in the network life cycle and the reconfiguration phase occurs in parallel with this stage [7].

Deeply analyzing, the knowledge areas considered to fit in the scope of activities held during operation and reconfiguration phases of construction projects are: 1) Performance Measurement; 2) Planning and Control; 3) Knowledge Management; 4) Resources Management; 5) Supply Chain Management (SCM), and; 6) Information and Communication Technologies (ICTs). But these activities are performed in a collaborative environment, with some discussion as follows.

Collaborative Networks in Civil Construction. A Collaborative Network is a group of autonomous heterogeneous entities geographically distributed (organizations and people) that have different operation environments, culture, capital, and targets to achieve common or compatible goals, with the support of computer networks [6]. VEs are kinds of Collaborative Networks. 
It is important to emphasize the difference between a Virtual Enterprise and a Virtual Organization. For the first one, the shared focus is the project at hand and the second one implies a conventional organization whose control is centralized [8]. The collaboration network approach of this paper is based on the temporary relations with focus on the project - construction project held as Virtual Enterprise. For the Performance Measurement of these VEs, the development of performance indicators based on the network benefits provides a tool to analyze the evolution of the collaborative processes and the individual performance of members [11]. The Management of Virtual Enterprises in terms of Planning and Control demands methods and models used for the coordination of the project activities. Initiatives as described in [5] and [12] can help at achieving collaborative planning strategies. Knowledge Management should provide effective sharing knowledge among operations with similar functions and geographically scattered [13], constitute ways and means of generating construction innovation [2] and improve communication and establish consistency among members, using a dedicated knowledge base [8]. But also very important is the capture and reuse of the project knowledge, that can be performed by post project reviews [13]. Human Resources are the main factor in Resource Management, dealing with the search for allocation of core competencies to achieve a culture for creation of knowledge value in which technological resources team work, capital, intellectual capital and change management - are mixed up according to the business needs [14]. For Supply Chain Management the trust among participants of the chain, between leaders and their teams [8] and also in the intra/inter-organizational dimensions [15] are of special importance. The chain operates in a dynamic environment in which agents, tasks and materials in process are strongly related to each other [16].

Information and Communication Technology - ICT, together with Knowledge Management are the most discussed issues when referring to transactions in collaborative environments. Several proposals, models, architectures have been developed to meet these requirements of Virtual Enterprises [6], [7], [17], [18], [5].

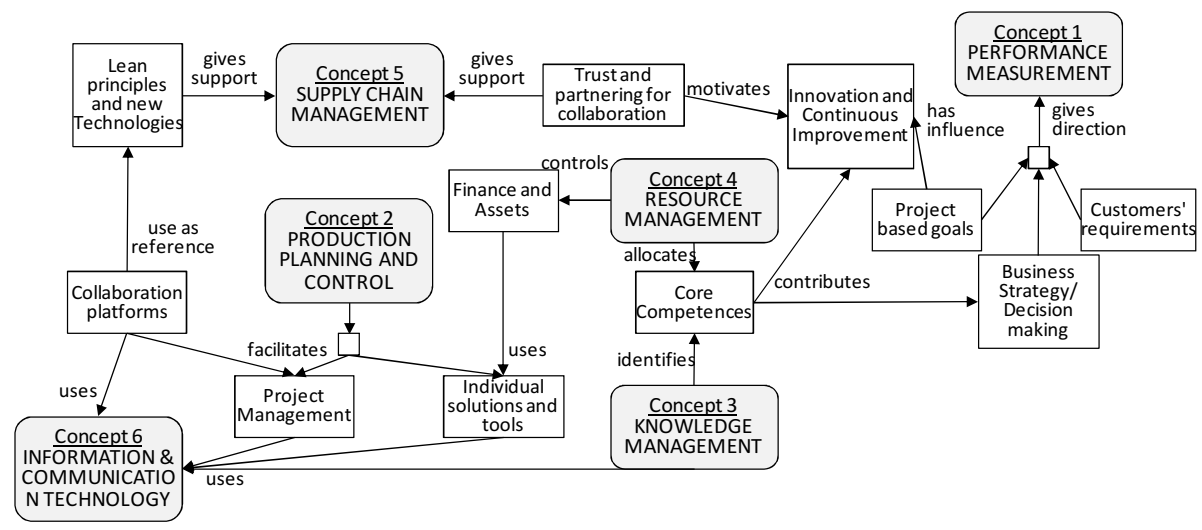

Fig. 1. Concept Model for construction collaborative networks: activities held during the operation and reconfiguration phases 
Fig. 1 shows how these discussed aspects are related, serving as a conceptual reference for the reference Model. It is emphasized that some papers were found giving references for the creation and dissolution phases for construction networks. Reference Models for the operation and reconfiguration phases of these networks were not found in literature. The study performed by [8] is a generic approach mostly based on knowledge management.

\section{$3 \quad$ Methodology}

As the main result desired by this research, a Reference Model is a standardized model recognized and approved by the interested parts and a model that can be used for the development or evaluation of other specific models [19]. For the elaboration of the Reference Model presented, the following steps were performed, based and adapted on [20] and [21], illustrated in Fig. 2. The first step is "Problem identification and planning". It involves the diagnosis of the problem observed in the research object (the network for the subway construction), definition of the modeling goals and planning of the Data Collection as a support for the next steps. The second step is the "Model Elaboration". Used for mapping the state of the art knowledge about the problem, delivering its reference framework (conceptual issues), then evaluated and refined. The third step is the "Reverse Analysis". It suggests a model elaboration from the perspective of the actual reality, delivering the As-Is Model. The fourth step is the "Definition of change" with a descriptive analysis of the changing possibilities from the As-Is Model to achieve the To-Be Model. The fifth and last step is the "Model Documentation" that contains a description of the represented Entities from the To-Be Model, linked to the possibilities identified in the changing definition and the modeling goals, delivering the Reference Model to be implemented.

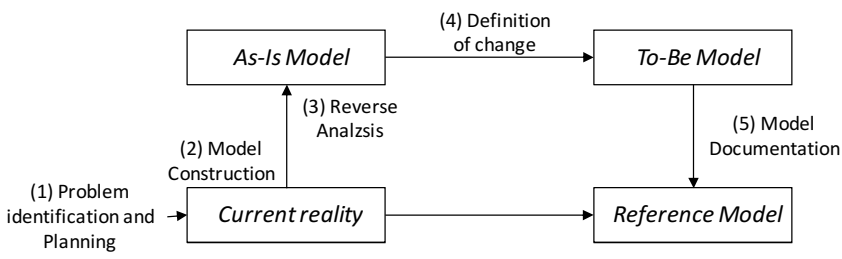

Fig. 2. Elaboration Process of the Reference Model.

The developed Models follow the EKD (Enterprise Knowledge Development) Methodology [22]. Three models from the methodology are used in this research - the Concept Model, the Actors and Resources Model and the Process Model.

The problem identification was stated in the introduction section and the conceptual reference is the result of the literature review. The development was done within a Case Study in a subway construction, following the literature indications [23]. This Case Study was the base for the current reality, the definition of change and the Reference Model further described. 


\section{Data Collection}

This section contains the description of the Subway Construction characteristics, its current reality and the change definitions base for the Reference Model developed.

The Subway Construction. The actor responsible for the subway construction is a Consortium of two big Brazilian Construction Companies. Both Companies have a wide know-how in infrastructure works. This Consortium is a strategic alliance that earned a concurrence process opened by the Government (state level). The concurrence stated the core competences desired, and only a few companies in Brazil were able to participate (all of them using the Consortium form). The final price was the criteria for the governmental choice. The Government is the main Consortium customer. The total construction will deliver twelve kilometers of subway in the most important metropolitan area in Brazil, with an initial budget of 10 billion dollars. It is a strategic construction due to integration possibilities with other public transportation options. The Consortium is responsible for the most expensive part of it.

The main phases for the construction performed are: 1) installation of construction site and support facilities, 2) trenching for equipment entrance and exit, 3) manufacturing of pre-fabricated components, 4) equipment operation for the tunnel opening (Shield), 5) excavation of wells and stations construction, 6) installation of permanent way and systems, 7) finishing and demobilization of equipment. Each part of these phases is a set of stages to be completed, demanding a wide variety of services and competences, part of them contracted by the Consortium - no more than $25 \%$ of the total budget. A current reality is described mostly discussing the processes and the actors and resources necessary to complete the stages.

Current Reality. Considering a processes perspective, each construction stage demands a proper "production order" to be initialized. To give this order, there is a common decision process applied to each stage during the construction execution. This decision process is mainly a set of checking issues: first it is checked the project's (design and specifications) completeness, second it is checked the availability of the actor/resource involved in that stage (including contracted partners) and third it is checked if the previous construction requirements are complete. For each decision loop, a contractual change can be necessary. The production order is given only if all checking issues have no problem and the contracts were changed (when necessary). This necessity for checking is mainly due to the poor quality of the projects (details and specification) and the poor quality of the plans - this second basically consequence of the first. The projects and macro plans are supplied by the Government. Through the time, this checking process leads to many reconfigurations of activities, actors and resources, and in the majority of cases, the project is delayed. It is necessary a good coordination between the process and the actors and resources involved in each stage.

The network was started by the Government, with the concurrence process. The two companies set the Consortium to construct with proper Resources (private capital) and Resources from the Government (public capital). The company responsible for the project was also contracted by the public entity. The Consortium subcontracts about another 50 companies for the most diverse services or supplying. 
It is necessary to emphasize that the most important criteria for verifying the core competences desired for the construction was the experience of the Engineers and the Companies in the construction of subways. Consultants are generally also contracted.

All actors are called Organizational Units (Government, Consortium, Companies, and Subcontracted Companies). Each Organizational Unit has at least one role to play inside the project. The Resources are also related to these Organizational Units.

Definition of Change. The Definition of Change is basically an association between the current reality verified and the conceptual reference stated by the literature review.

To assist the performance measurement, it is necessary to consider the final user of the construction as the main customer - the perspective of the business strategy. The measured issues can be used for decision making. Partnering, collaboration and trust must be encouraged among actors, as a requisite for knowledge management. Also, for planning and control, the individual solutions used by each network actor can have interface with a common system to ensure the information, project, plans and knowledge sharing. A collaboration platform can be seen as an appropriate ICT functionality, influencing also the resource and supply chain management.

\section{$5 \quad$ The Reference Model}

The Reference Model is composed of two sub-models. The Process Model (Figure 3) represents the process for decision making within the operation and reconfiguration phases (having trust as the main background), and minimizing the necessity of checking and changing contracts. It can also be considered an activity model, considering the flow of activities performed during the construction. The important reference is the organization of construction phases, not the decision flow. As an actual reality was the main approach to be improved, the decision making process was the main focus.

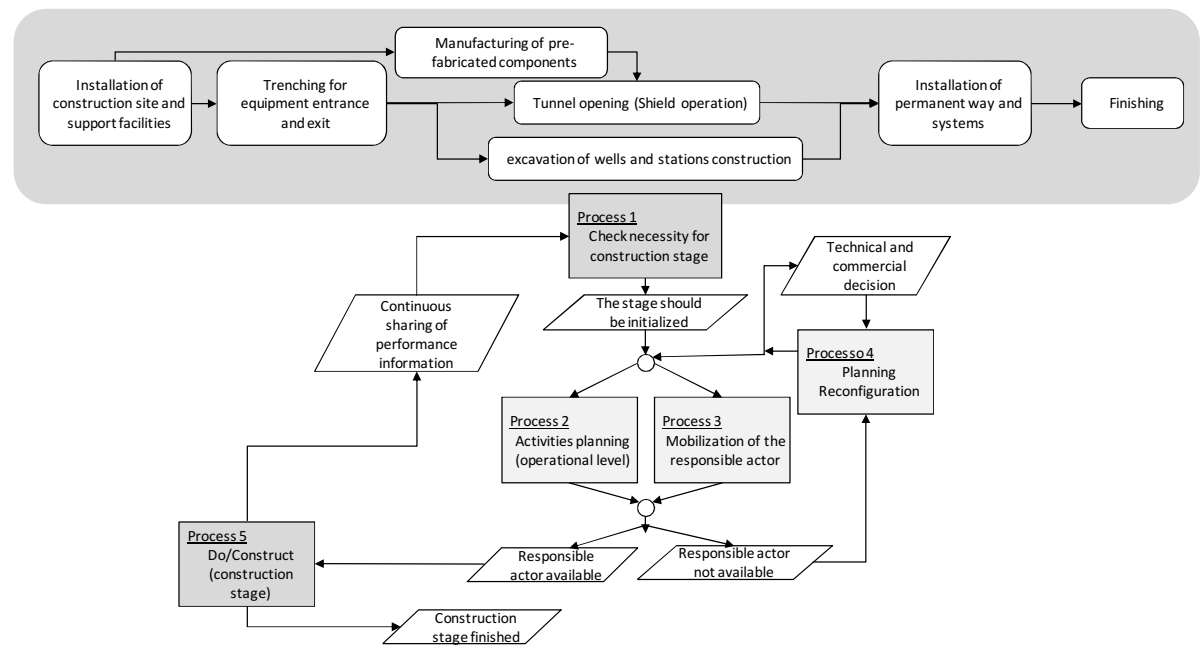

Fig. 3. Process Model for operation and reconfiguration of a network for subway construction 
The Actors and Resources Model (Figure 4) represents a generalist framework reflecting the requirements discussed in the Definition of Change. It states clearly the consolidation of the Virtual Enterprise, the collaborative emphasis supported by the collaboration platform and the discussed issues for operation and reconfiguration of these networks.

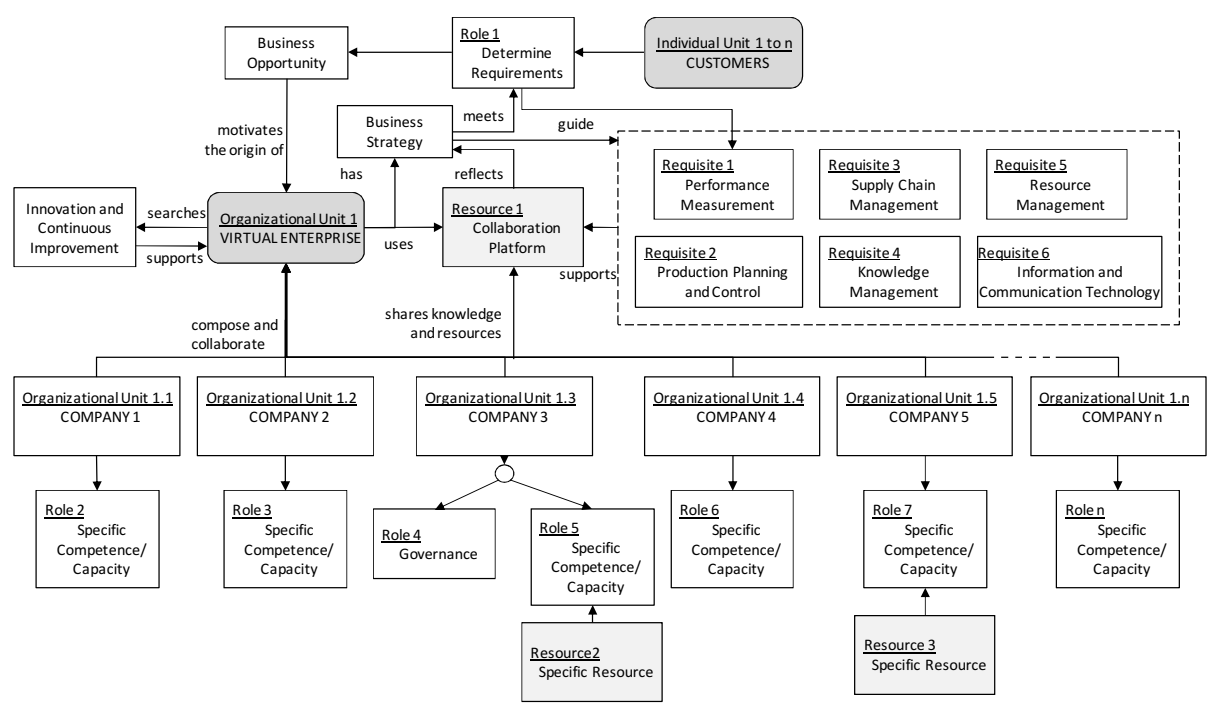

Fig. 4. Actors and resource model for operation and reconfiguration of a network for subway construction

\section{Conclusion}

The Reference Model contributes to the operation and reconfiguration phases of construction networks, especially those of large scale projects. It denotes a better allocation of actors and resources, supporting a better coordination and organizational control - the main challenges for managing the large construction projects.

The operation and reconfiguration phases, considering collaborative networks, still demands practical and academic efforts to compose the agenda of managers and researchers. It is necessary to focus on the decision making processes' quality to enhance efficacy and effectiveness of projects and networks. The absence of qualified personnel for managing large scale projects was verified within the Case Study. The allocation of competences based only in experience as seen, decreases the possibility of value enhancing by innovation and continuous improvements for these projects.

The Reference Model development assists the knowledge systematization. The aim is to identify aspects, practices, configurations that can be replied to other subway constructions, serving as reference for the public and private interested organization/institution. It can be used as a new approach for Public Management. It demands a paradigm disruption in terms of configuration and contractual relations, requiring the "trust" as a principle inside public projects. 
The model can be adapted to other construction works of infrastructure (bridges, roads, stadiums, dams), considering its proper requirements for configuration, its own processes and the network characteristics. This model presented has the limitation of being based only in one case study. As no previous reference models for operation and reconfiguration phases of construction networks were found in literature, it is also not possible to make any comparisons. This is the reason it is considered an initial effort.

\section{References}

1. Lee, S.H., Peña-Mora, F., Park, M.: Dynamic planning and control methodology for strategic and operational construction project management. Automation in Construction 15, 84-97 (2006)

2. Rutten, M.E.J., Dorée, A.G., Halman, J.I.M.: Innovation and interorganizational cooperation: a synthesis of literature. Construction Innovation 9(3), 285-297 (2009)

3. Yu, H., Tweed, T., Al-Hussein, M., Nasseri, R.: Development of lean model for house construction using value stream mapping. Journal of Construction Engineering and Management 135, 782-790 (2009)

4. Tanskanen, K., Holmström, J., Elfving, J., Talvite, U.: Vendor-managed-inventory (VMI) in construction. Int. Journal of Productivity and Performance Management 58, 29-40 (2009)

5. Keller, M., Scherer, R.J., Menzel, K., Theling, T., Vanderhaeghen, D., Loos, P.: Support of collaborative business process networks. In: AEC, ITcon. 11, Special Issue Process Modelling, Process Management and Collaboration, pp. 449-465 (2006)

6. Camarinha-Matos, L., Afsarmanesh, H.: Collaborative networks: a new scientific discipline. J. Intelligent Manufacturing 16, 439-452 (2005)

7. Camarinha-Matos, L., Afsarmanesh, H.: On reference models for collaborative network organizations. Journal of Production Research 46, 2453-2569 (2008)

8. Rezgui, Y.: Exploring virtual team-working effectiveness in the construction sector. Interacting with Computers 19, 96-112 (2007)

9. Vorakulpipat, C., Rezgui, Y., Hopfe, C.J.: Value creating construction virtual teams: A case study in the construction sector. Automation in Construction 19, 142-147 (2010)

10. Rahman, M.M., Kumaraswamy, M.M., Ling, F.Y.Y.: Building a relational contracting culture and integrated teams. Canadian J. of Civil Engineering 34(1), 75-88 (2007)

11. Camarinha-Matos, L., Abreu, A.: Performance indicators for collaborative networks based on collaboration benefits. Production Planning \& Control 18, 592-609 (2007)

12. Verheij, H., Augenbroe, G.: Collaborative planning of AEC projects and partnerships. Automation in Construction 15, 428-437 (2006)

13. Dave, B., Koskela, L.: Collaborative knowledge management - A construction case study. Automation in Construction 18, 894-902 (2009)

14. Cheng, M.Y., Tsai, M.H., Xiao, Z.W.: Construction management process reengineering: Organizational HR planning for multiple projects. Aut. in Construction 15, 785-799 (2006)

15. Zou, P.X.W., Zhang, G., Wang, J.: Understanding the key risks in construction projects in China. International Journal of Project Management 25, 601-614 (2007)

16. Landesmann, M., Scazzieri, R.: Production and economic dynamics. Cambridge Press (1996) 
17. Camarinha-Matos, L., Afsarmanesh, H.: Elements of a base VE infrastructure. Journal of Computers in Industry 51, 139-163 (2003)

18. Goranson, H.T.: The Agile Virtual Enterprise: Cases, Metrics, Tools. Quorum Books, Westport (1999)

19. Vernadat, F.B.: Enterprise Modeling and Integration: Principles and Applications. Chapman \& Hall, London (1996)

20. Ahlemann, F., Gastl, H.: Process model for an Empirically Grounded Reference Model Construction. In: Fettke, P., Loos, P. (eds.) Reference Modeling for Business Systems Analysis, pp. 77-97. Idea Group Publishing, Hershey (2007)

21. Nurcan, S., Rolland, C.: A multi-method for defining the organizational change. Information and Software Technology 45(2), 61-82 (2003)

22. Bubenko Jr., J.A., Brash, D., Stirna, J.: EKD User guide. Department of Computer and Systems Sciences. Royal Institute of Technology, Stockholm (2001)

23. Yin, R.K.: Case study research: Design and methods, 3rd edn. Sage, California (2003) 